\title{
Aspects of AMnet Signaling
}

Anke Speer, Marcus Schöller, Thomas Fuhrmann, and Martina Zitterbart

Universität Karlsruhe, Germany

\begin{abstract}
AMnet provides a framework for flexible and rapid service creation. It is based on Programmable Networking technologies and uses active nodes (AMnodes) within the network for the provision of individual, application-specific services. To this end, these AMnodes execute service modules that are loadable on-demand and enhance the functionality of intermediate systems without the need of long global standardization processes.

Placing application-dedicated functionality within the network requires a flexible signaling protocol to discover and announce as well as to establish and maintain the corresponding services. AMnet Signaling was developed for this purpose and will be presented in detail within this paper.
\end{abstract}

Keywords: Programmable Networks, Active Nodes, Multicasting, Signaling

\section{Introduction}

Many new evolving Internet applications are based on one-to-many or manyto-many communication, e.g., tele-teaching, tele-collaboration, information dissemination through push technologies, and web-radio. IP multicast 2 efficiently supports this type of transmission with a receiver-oriented concept: receivers join a particular multicast session group and traffic is delivered to all members of that group by the network infrastructure.

A challenge that comes with this type of communication is the possible heterogeneity in the group members' service requirements. These may vary dependent on the individually available performance of the network access, the type of end system being used, the willingness to pay a higher price for better quality of service and the like. Today, most approaches realizing heterogeneous group communication adjust the provided data stream for all group members according to the group member with the lowest performance. This, however, is not desirable for many multimedia or distributed applications (e.g., video conferencing, gaming).

Besides group communication applications, also other Internet applications benefit from additional network support (e.g., management or control facilities). However, introducing new functionality into the network has to be in-line with new evolving applications for realizing proper communication support promptly. Unfortunately, progress in supporting new network functionality is very slow

E. Gregori et al. (Eds.): NETWORKING 2002, LNCS 2345, pp. 1214-1220 2002.

(C) Springer-Verlag Berlin Heidelberg 2002 
because the current network infrastructure is inflexible. The introduction of new services and protocols to enhance network functionality typically requires long global standardization processes.

AMnet addresses rapid service creation in the context of heterogeneous group communication to allow the flexible and rapid introduction of new functionality in global networks. AMnet is based on Programmable Networking technologies and aims at building an implicit overlay network on top of the existing IP infrastructure for the completion of application-specific requirements. According to the Programmable Networking approach, so-called service modules are installed on active intermediate nodes - the AMnodes. AMnodes form the core building blocks of AMnet and operate on the multicast distribution tree used for the communication between sender and receivers. Service modules are responsible for the adaptation of data streams to specific service demands [5].

This paper is structured as follows: The next section presents the developed inter-domain signaling protocol for AMnet in detail. Section 2.1 describes the management of different services within AMnet, followed by the mechanisms of the establishment and maintenance of these services in Section 2.2. In Section 2.3. the way a receiver is provided with its dedicated services is presented. Section 2.4 lays special focus on the new concepts developed for evaluating AMnodes to determine their capabilities as possible service providers. The paper closes with a summary and an outlook on future work.

\section{Concepts of AMnet Signaling}

AMnet aims at dynamically placing application-specific functionality within the network. To this end, some questions have to be decided: how should different services be managed within a session, how should they be established and maintained, how should a receiver be associated to a dedicated service and where should those services be placed? In this context, a session describes a communication scenario where a designated sender issues a data stream which can be received from several communication participants without or after adaptation in the AMnodes. To solve the foregoing questions a flexible and light-weight signaling protocol for AMnet was developed [6] which will be presented in the following section.

\subsection{Management of Services}

Service heterogeneity within a session needs to be bound to a manageable degree of diversity. Therefore, one concept of AMnet signaling is to logically group receivers with similar service demands into distinct multicast receiver groups - the service level groups - distinguishable by their multicast-addresses. The receivers join the corresponding group on demand through IGMP 3 .

Each service level group within a communication session represents all receivers whose service demands can be satisfied with a single group service. 
Therefore, each group represents a different view onto the same original data corresponding to the adaptation performed by the AMnodes.

The service of a group is supported by an AMnode through the use of appropriate service modules. The actual service is then derived from the processing of the original data stream (cf., AMnode 2 in Figure 1) or from the service of another service level group (cf., AMnode 3 in Figure 1). Therefore, the communication service offered by AMnet can be described by a tree of service level groups (cf., Figure 1).

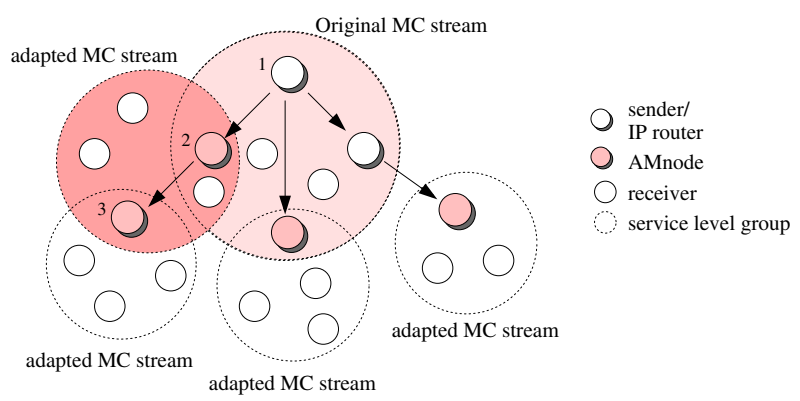

Fig. 1. Multicast Tree with Service Level Groups

\subsection{Establishment and Maintenance of Services}

Service modules are held in distributed data bases - the service module repositories - which are administratively managed per domain. Service modules can be stored there by trusted AMnet users or network management procedures. Moreover, current work focuses on establishing a hierarchy of trusted repositories. The stored service modules are grouped into module classes like, e.g., audio transcoding or reliable multicast, and for each module class there exists a distinct evaluation procedure to be downloaded within the evaluation process (cf., Section 2.4). Therefore, the overall purpose of the repositories is to make service modules and their corresponding evaluation procedures available to an AMnode which is requested to provide a special service (cf., Figure 2 (4)).

Besides multicasting the original data stream of the session (cf., Figure 2 (1)), the sender announces the provided session on a separate multicast group - the session control group (cf., Figure 2 (1a)). In this group every AMnet session is announced similar to the Session Announcement Protocol of the MBone [4]. The session announcement contains a description of the session including bandwidth and delay requirements, as well as content specific information like data format and compression scheme. This description is used by the potential session participants to determine in which way the original data stream has to be adapted by the AMnodes to receive the data stream at a desired service level. 
Moreover, the description contains the multicast address of the original data stream and the multicast address of the service announcement group. In this group, the AMnodes announce the provided services (cf., Figure 2(5)). Potential session participants join the service announcement group to learn about available services (cf., Figure 2 (3)), i.e., the description of the provided service, as well as the address of the corresponding service level group where the adapted data is sent to. Moreover, during multicast distribution the address of each AMnode that was traversed by the announcement on its way from the sender to the receiver is included. This information is used for the evaluation process described in Section 2.4.

Service modules on an AMnode are maintained in a soft state. After joining a service level group the participant periodically sends HELLO messages to the AMnode hosting the appropriate service module. If no HELLO messages were received for a given time, the AMnode makes the service module stop issuing data into the corresponding service level group. However, the service modules are not immediately deleted from the AMnodes but cached, in case the service is requested again right afterwards. The soft state of the service modules is utilized to prevent service level groups not used by any participants. The caching strategy helps to avoid unnecessary overhead coming along with re-loading and re-installation.

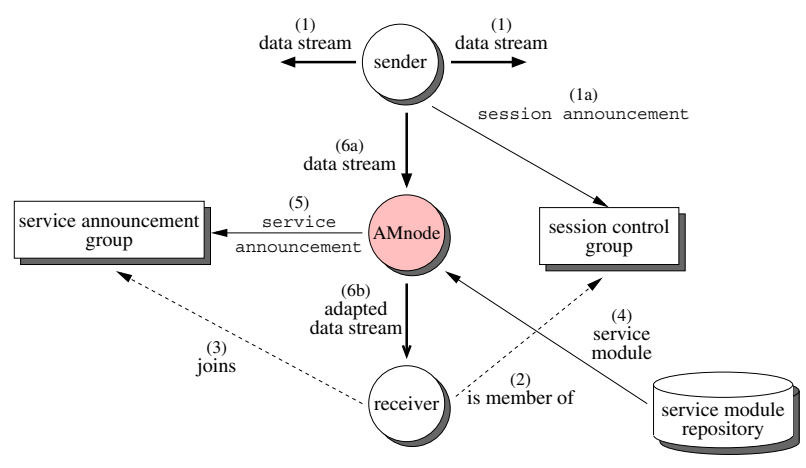

Fig. 2. Overview of the AMnet Service Control

\subsection{Association between Receiver and Service}

A receiver that wants to use a special service for adapting the data stream to its requirements processes the service announcements. If one of the announcements matches the receiver's requirements, it simply joins the corresponding service level group where the adapted data stream is sent to.

Otherwise, if no matching service is announced, an evaluation process has to be started (cf., Section 2.4). Within this process, AMnodes on the data path 
between the sender and the receiver are analyzed whether to be capable of providing the desired service. The AMnodes on the data path are known from the corresponding service announcements of the sender that provides the data stream the receiver wants to be adapted (cf., Section 2.2). Even if no additional service is advertised in the service announcement group, at least the service of the session sender providing the original data stream is announced.

The evaluation process will result in the address of an AMnode that is considered to be a good place for supporting the service. Then, the appropriate service module is downloaded from the service module repository onto this AMnode (cf., Figure 2 (4)). For security reasons, the service modules will be signed and only modules with a correct signature will be installed on an AMnode. The newly installed service is announced into the service announcement group (cf., Figure 2 (5)) and the receiver can simply join the corresponding service level group. Now, it will experience the data stream adapted according to its requirements (cf., Figure 2 (6a)-(6b)).

\subsection{The Evaluation Process}

In the original approach of the evaluation process for AMnet [6] the intra-domain evaluation of the AMnodes was realized with active evaluation packets corresponding to capsules as introduced in the Active Networking context [1. These capsules contained an evaluation program downloaded from the service module repository and initialized by the receiver. This approach, however, was not usable within the context of inter-domain signaling because of security considerations. Therefore, a new approach was developed. Now, the receiver has only to issue a service request to its predecessor AMnode (cf., Figure 3) known by the path information of the service announcements. The service request contains the class of the service the receiver wants to be supported (e.g., audio transcoding) and the path information. Moreover, specific parameters can be included. In the case of audio transcoding, this may be the maximum data rate the receiver is able to process, the formats of the data stream the receiver can understand, and so on.

The first AMnode that is able to process the receiver's service request contacts the service module repository and downloads the evaluation procedure that corresponds to the module class the desired service belongs to. With this evaluation procedure the AMnode is analyzed. The results of the local evaluation, the address of this local AMnode, as well as the identifier for the used evaluation procedure are tied up into an evaluation packet that is forwarded to the next AMnode (cf., Figure 3) known from the path information contained in the service request. Moreover, this path information is included into the packet, as well as the address of the first AMnode that processed the receiver's service request and started the whole evaluation process.

An AMnode that receives an evaluation packet contacts its responsible service module repository to download the specified evaluation procedure. The AMnode will contact the same service module repository for downloading a requested service. Therefore, if the specified procedure is not included in the contacted service module repository, the AMnode will not be able to support the desired 
service at all, because service class and corresponding evaluation procedure are always stored as one entity in a repository entry. Then, the evaluation packet will only be forwarded unchanged to the next AMnode on the path towards the session sender. However, if the contacted service module repository contains the specified evaluation procedure, it will be downloaded and executed on the AMnode as described above. Evaluation results are only entered into the evaluation packet if the local AMnode fulfills the given requirements in a better way than the AMnode already registered in the packet. After the evaluation procedure is finished on the local AMnode, the evaluation packet is forwarded to the next AMnode, again. This process stops on the last AMnode in front of the session sender. After its evaluation, the final evaluation packet is sent back to the first AMnode that started the whole procedure (cf., Figure 3). This AMnode, now, interprets the evaluation result as the address of the AMnode that is considered to be the best for supporting the service in the current network scenario. This AMnode, then, is made to download and install the requested service module and the receiver can access the desired service level as described in Section 2.3 .

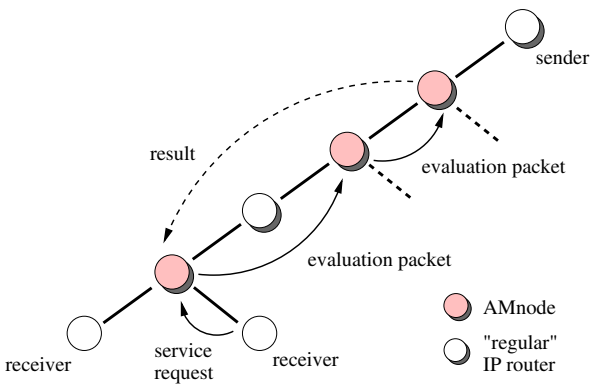

Fig. 3. Scheme of the Evaluation Process

\section{Conclusions and Outlook}

AMnet provides an open and generic framework for the provision of user-tailored rapid service creation with a specific focus on heterogeneous group services. It is based on Programmable Networking technologies and aims at building an overlay network on top of the available Internet infrastructure. A major goal of AMnet is to provide individual services on demand without complex installation and management overhead. AMnet is based on IP and benefits from its multicast extensions in several ways. For realizing service and session announcements, as well as for disseminating adapted data with individual requirements distinct IP multicast groups are applied. 
This paper is focused on AMnet Signaling - a flexible and active signaling protocol - developed specifically to support the placement and announcement as well as the establishment and maintenance of active services in the context of rapid service creation with AMnet. Moreover, the new developed evaluation process for placing dedicated services inside the network was described. In contrast to the mechanisms presented in [6], this evaluation mechanism can be used inter-domain.

Future work will focus on extending or, respectively, changing the signaling mechanisms to be able to use AMnet as well in networking environments where native IP Multicast is not provided. Different approaches are considered and will be evaluated. Moreover, the presented novel evaluation process will be introduced in the actual prototype implementation, leading to enhanced experience with automated service discovery and placement.

\section{References}

1. J. V. Guttag D. J. Wetherall and D. L. Tennenhouse. ANTS: A Toolkit for Building and Dynamically Deploying Network Protocols. In Proceedings of the IEEE OPENARCH, pages 117-129, April 1998.

2. S. Deering. Host Extensions for IP Multicasting. RFC 1112, IETF, August 1994.

3. B. Fenner. Internet Group Management Protocol, Version 2. RFC 2236, IETF, November 1997.

4. M. Handley. SAP: Session Announcement Protocol. Internet draft, IETF, November 1996.

5. T. Harbaum, B. Metzler, R. Wittmann, and M. Zitterbart. AMnet: Heterogeneous Multicast Services based on Active Networking. In Proceedings of the IEEE OPENARCH99, pages 98-107, New York, NY, USA, March 1999. IEEE.

6. A. Speer, R. Wittmann, and M. Zitterbart. Locating Services in Programmable Networks with AMnet Signalling. In Proceedings of The Sixth Conference on Intelligence in Networks (SmartNet 2000), Wien, Austria, September 2000. 